\title{
Evaluation of MRI Orthogonal Planes in Differential Diagnosis of Non-Tumour Brain Lesions
}

ABSTRACT

\section{BACKGROUND}

Magnetic resonance imaging (MRI) provides structural characterization of brain lesions, by measuring volume of axial, sagittal and coronal planes through two dimensional slices. The purpose of this study was to characterize and identify the orthogonal imaging planes to detect non tumour lesions of brain through MRI.

\section{METHODS}

This study included 81 patients, both males and females, suspected of brain lesions and underwent MRI for diagnosis. The variations in the volume of the anatomical structures were measured and compared the planes as axial and sagittal, axial and coronal and coronal and sagittal for non-tumour brain lesions.

\section{RESULTS}

The present study revealed the differences in the measurement of volume in nontumour lesions $(\mathrm{N}=81)$ in axial, sagittal and coronal planes. It was found that the volume of axial planes (9.2) is more dominant than the sagittal (9.1) and coronal planes (8.8) in non-tumour lesions. Statistical analysis was done by Statistical Package for Social Sciences (SPSS version 16 software). Two way/Friedman test were used for comparing the three groups.

\section{CONCLUSIONS}

This study concluded that, in most of the brain lesions irrespective of the type of tumours, axial planes helps more in the detection of tumour volume as compared to sagittal and coronal planes for precise diagnosis of brain lesions.
Corresponding Author: Smitha H.,

Sree Gokulam Medial College and Research Foundation, Venjaramoodu, Trivandrum, Kerala, India.

E-mail: smithavinod2000@yahoo.co.in

DOI: $10.14260 / j e m d s / 2021 / 718$

How to Cite This Article:

Smitha H, Devi VNM, Sreekanth KS, et al. Evaluation of MRI orthogonal planes in differential diagnosis of non-tumour brain lesions. J Evolution Med Dent Sci 2021;10(40):3543-3547, DOI: 10.14260/jemds/2021/718

Submission 04-03-2021, Peer Review 18-09-2021, Acceptance 25-09-2021, Published 04-10-2021.

Copyright (C) 2021 Smitha H. et al. This is an open access article distributed under Creative Commons Attribution License [Attribution 4.0 International (CC BY 4.0)]

\section{KEY WORDS}

Axial Plane; Coronal Plane; Magnetic Resonance Imaging; Non-Tumour Brain Lesions; Sagittal Plane. 


\section{BACKGROUND}

Magnetic resonance imaging provides excellent soft tissue delineation of different areas of interest. It is established for both diagnosis and research purpose. ${ }^{1}$ MRI is a sensitive tool for the diagnosis of normal, abnormal and surrounding structures of brain. ${ }^{2}$ In modern neuroimaging, MRI permits superior structural characterization by diagnosing the cellular, vascular, metabolic and functional properties of brain tumors. ${ }^{3}$ It uses nonionizing radiation and provides 3-dimensional reconstruction of organs from multiple contiguousimage. ${ }^{4}$ Surface coils, fat suppression techniques, fast gradient-echo pulse sequences are the modern technical developments. Excellent spatial and contrast resolution of the soft tissues are provided by MR contrast agents with direct multi planar imaging. 5 Through the human body, MRI can produce thin slice image. MRI is based on the absorption and emission of energy in the radiofrequency range of the electromagnetic spectrum which is an advanced technique. ${ }^{6}$

The hydrogen $(\mathrm{H})$ nuclei are used for MR imaging, as the MR imaging is based on the electromagnetic activity of atomic nuclei. ${ }^{7}$ The net magnetization produced by the protons of human body, will align parallel to the main magnetic field which is used to produce MR images. ${ }^{8}$ TR (repetition time) is the time between RF pulses. TE (echo time) is the time between initial RF pulse and the acquisition signal. Short TR and short TE produce the T1 weight (wt) images, provides the anatomical detail, but long TR and long TE produce T2 wt images. ${ }^{9}$ It was found that the evaluation of signal intensity of brain lesions of both T1wt images and T2 wt images also have a significant role to find out the early diagnosis of tumour as well as non-tumour lesions. 10

The coordinate system of MRI is represented as $\mathrm{x}, \mathrm{y}, \mathrm{z}$, the longitudinal direction is given as $\mathrm{Z}$ direction (parallel to the main magnetic field). The plane perpendicular to the main magnetic field is called $x-y$ plane. A patient, who is headfirst and supine, the $\mathrm{x}$ direction is chosen to be left -right direction and the anterior posterior direction as y direction. The axial plane matches the transverse plane. ${ }^{8} \mathrm{MRI}$ is capable of producing high resolution thin tomographic images in any plane and blocks of 3-dimensional information and has no known biological hazard. ${ }^{11}$

The three standard anatomical planes are axial, sagittal and coronal planes. Any vertical plane parallel to the median plane is referred as sagittal plane. A vertical plane perpendicular to the sagittal plane is the coronal plane. Whereas the axial plane is perpendicular to both sagittal and coronal plane. ${ }^{12}$ The brain can be viewed in three perspectives in MRI, they are transverse (axial or x-y) plane, which slice the patient from top to bottom whereas sagittal $(y-z)$ planes slice the patient laterally and coronal plane (x-z) slice the patient from front to back. In the brain MRI volume, axial, sagittal and coronal planes consist of two-dimensional slices. ${ }^{13}$ MRI also provides detailed information which helps in differential diagnosis and management of brain lesions. ${ }^{14}$ Since MRI planes play an important role in imaging of brain lesions, the aim of the study is to characterize and identify the orthogonal imaging planes to detect non-tumour brain lesions through MRI.

\section{METHODS}

This study was an observational study, which was conducted in the Radiology Department of one of the major tertiary health care centres in South Kerala, India from 2018 November to 2019 October. Patients suspected of brain lesions and who underwent MRI for diagnosis were included. Approval was obtained from The Institutional Ethics Committee. In this study, both males and females were included. Patients with the proven MRI diagnosis were only included as study subjects. Those with implants, aneurysm clips, pacemakers, heart valves and those who are not willing to participate in the study and patients with age below 20 years were excluded from this study.

MR imaging was done for patients with different brain lesions including non-tumour lesions $(\mathrm{N}=81)$. With a $1.5-\mathrm{T}$ scanner, imaging was performed and studied for their differential diagnosis through different planes (axial, coronal and sagittal) with a head coil of diameter $30 \mathrm{~cm}$. With the help of sophisticated combination of electronics, radiofrequency (RF) generators, coils, and gradients, the MR images were generated that interface with a computer for communication between the different electronics. This combination of equipment allows localization, excitation, and acquisition of a specific tissue of interest and formation of a digital image. The specialized equipment in MRI like gradient coils, magnets and radiofrequency coils generates and receives the MR signal. The protons align to the main field $\left(\mathrm{B}_{0}\right)$ in the $\mathrm{z}$ direction, when the subject is placed into a magnetic field and the Larmor frequency is field dependent (e.g., Larmor frequency $\omega=\gamma B_{0}$, where $\gamma=42.6 \mathrm{MHz} / \mathrm{T}$ and is called the gyromagnetic ratio). MR signal is localised by applying a gradient that produces a controlled linear spatial variation of the Bomagnetic field ( $\mathrm{z}$ direction), which creates small perturbations to the field in three directions ( $\mathrm{x}, \mathrm{y}$, and $\mathrm{z}$ ). The three functions like slice selection ( $\mathrm{z}$ component), frequency encoding ( $\mathrm{x}$ component), and phase encoding (y component) were performed by the gradient coils. The axial plane is referred as the slice perpendicular to the $\mathrm{z}$ axis. The plane that divides the brain into left and right parts is called sagittal or median planes. The vertical plane, which divides the brain into posterior and anterior parts is called coronal plane. ${ }^{15}$

By using spin-echo pulse sequences, gradient echo sequences, FLAIR and a two-dimensional Fourier transform image reconstruction all images were obtained. T1 - wt spinecho images $(500-600 / 20-30 / 2$ - 4 repetition time / echo time / excitations) and T2 - wt spin-echo, FLAIR, gradient echo (2500-3000 / 30, 80 / 1) were obtained from all patients. The images were acquired on a $256 \times 256$ matrix, with a field of view of $23 \mathrm{~cm}$. All images had a slice thickness of $5 \mathrm{~mm}$ and the number of slices taken was 20. The MRI studies were evaluated for the volume of brain lesions on three different planes. A reference line was drawn as the longest tumour diameter anteroposteriorly on the axial view, mediolaterally on the coronal view, and superio-inferiorly on the sagittal view respectively. Figure 1, Figure 2, Figure 3, shows non tumour brain lesion in axial plane, sagittal plane and coronal plane for same non tumour. Size of the brain lesions were measured including length and width. Volume was also calculated by using the formula. ${ }^{16}$ 
Volume $=\frac{\text { Area of the brain lesion } x \text { Slice thickness }}{\text { Number of Slices }}$
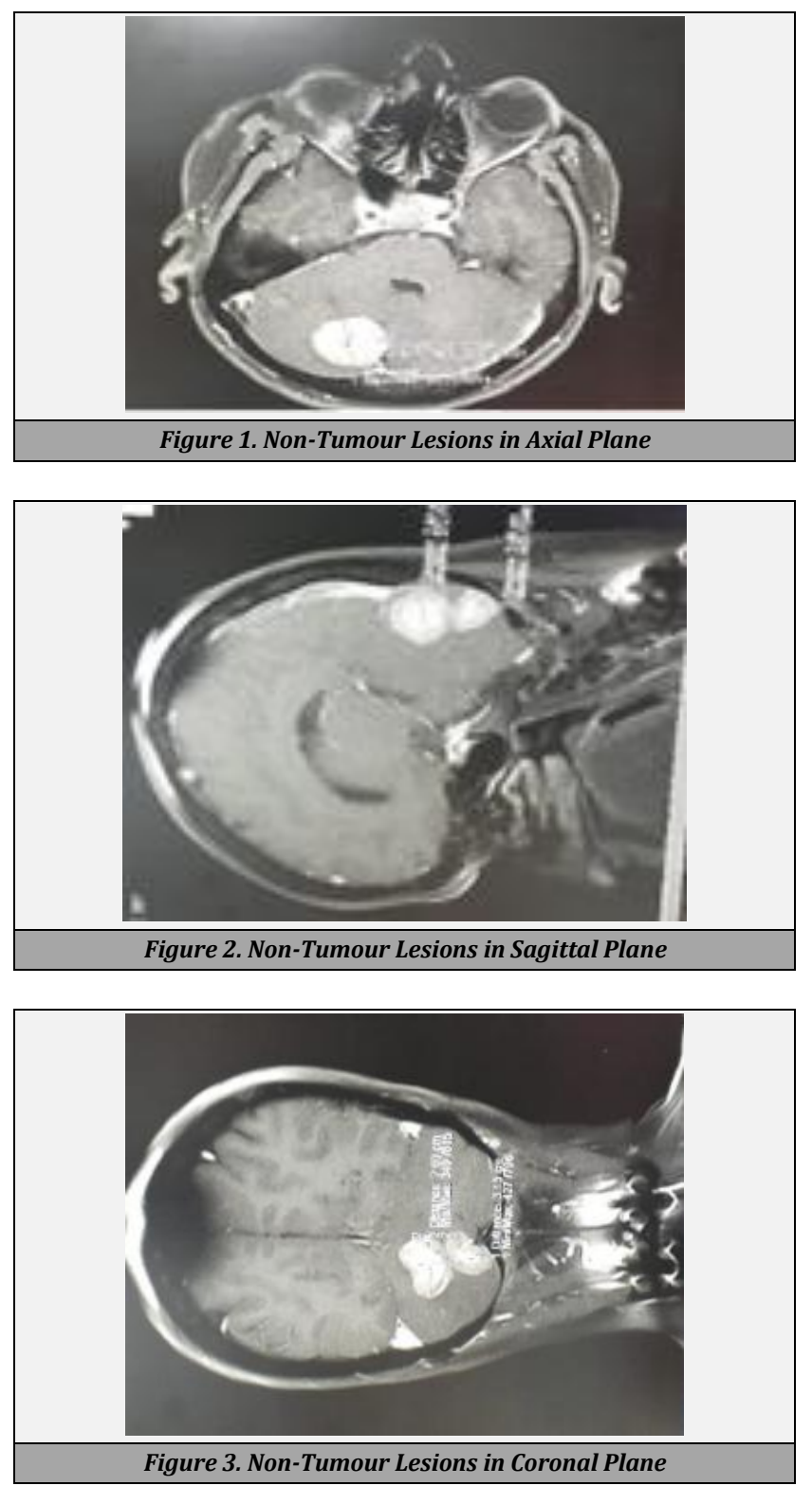

For this study, the non-tumour brain lesions $(\mathrm{N}=81)$ were only selected. Depending on the volume dimension, the nontumour lesions were categorised as group I, II, III, IV, V, VI, VII, VIII and IX. Group $1(\mathrm{~N}=22)$ indicates the dimensions from 0.1 $\mathrm{mm}$ to $0.9 \mathrm{~mm}$ whereas group II $(\mathrm{N}=12)$ gives the dimensions from $1 \mathrm{~mm}$ to $1.6 \mathrm{~mm}$, group III ( $\mathrm{N}=7$ ) from $2.0 \mathrm{~mm}$ to $3.9 \mathrm{~mm}$ group IV ( $\mathrm{N}=5)$ from $4 \mathrm{~mm}$ to $4.9 \mathrm{~mm}$, group $\mathrm{V}(\mathrm{N}=8)$ from $5.8 \mathrm{~mm}$ to $8.0 \mathrm{~mm}$, group VI $(\mathrm{N}=6)$ from $9.5 \mathrm{~mm}$ to $11.5 \mathrm{~mm}$, group VII $(\mathrm{N}=6)$ gives $15.5 \mathrm{~mm}$ to $16.5 \mathrm{~mm}$, group VIII $(\mathrm{N}=$ 6) $15 \mathrm{~mm}$ to $18 \mathrm{~mm}$ and group IX ( $\mathrm{N}=9$ ) from $21.4 \mathrm{~mm}$ to 23.8 $\mathrm{mm}$. The variations in the volume of the anatomical structures were measured and compared the planes like axial and sagittal, axial and coronal and coronal and sagittal planes.

\section{Statistical Analysis}

Statistical analysis was done by SPSS version 16 software. The quantitative variables were expressed in mean and standard deviation (SD). Two way/Friedman test was used for comparing the three groups.

\section{RESULTS}

In this study, the non-tumour lesions $(\mathrm{N}=81)$ like neurocysticercosis, meningitis, abscess, subarachnoid haemorrhage, subdural haematoma, multiple sclerosis, cerebral ischemia, Fazekas grade 1, Fazekas grade 2, Fazekas grade 3, cerebral infract, epidermoid cyst, arachnoid cyst, mastoiditis, and cystic lesion were included. Table 1 shows the volume measurement of non-tumour lesions which are grouped as I, II, III, IV, V, VI, VII, VIII and IX. It was found that among the 9 groups of non-tumour lesions, the average volume of 6 groups (group II, IV, VI, VII, VIII, IX) in axial planes was more superior than in sagittal and coronal planes. The other non-tumour lesions in group I, group III and group V, the coronal plane was determined as superior as other two planes.

\begin{tabular}{|c|c|c|c|c|c|c|c|c|c|}
\hline \multicolumn{10}{|c|}{ Non Tumour Volume Measurements of MRI Orthogonal Planes } \\
\hline \multirow[t]{2}{*}{ Groups } & \multicolumn{3}{|c|}{ Axial Plane } & \multicolumn{3}{|c|}{ Sagittal Plane } & \multicolumn{3}{|c|}{ Coronal Plane } \\
\hline & $\begin{array}{l}\Sigma \\
\sum \\
\Sigma\end{array}$ & 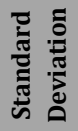 & $\underbrace{\stackrel{0}{ٍ ~}}_{0}$ & 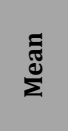 & 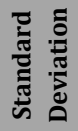 & 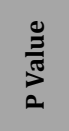 & 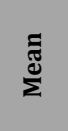 & 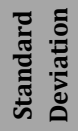 & 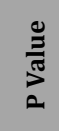 \\
\hline I & 0.55 & 0.357 & 0.004 & 1.24 & 1.82 & 0.004 & 1.05 & 1.43 & 0.004 \\
\hline II & 1.39 & 0.194 & 0.472 & 1.37 & 0.262 & 0.472 & 1.31 & 0.23 & 0.472 \\
\hline III & 2.93 & 0.905 & 0.097 & 2.98 & 1.019 & 0.097 & 2.72 & 0.84 & 0.097 \\
\hline IV & 4.47 & 0.333 & 0.165 & 4.4 & 0.235 & 0.165 & 4.06 & 0.39 & 0.165 \\
\hline $\mathrm{V}$ & 7.80 & 1.171 & 0.197 & 8.3 & 6.44 & 0.197 & 8.33 & 1.56 & 0.197 \\
\hline V I & 10.19 & 1.069 & 0.019 & 9.40 & 1.316 & 0.019 & 9.13 & 1.09 & $0.01 \mathrm{~s}$ \\
\hline V II & 16.24 & 0.517 & 0.002 & 15.72 & 0.507 & 0.002 & 15.25 & 0.92 & 0.002 \\
\hline VIII & 16.64 & 1.37 & 0.115 & 17.09 & 0.933 & 0.115 & 16.19 & 1.00 & 0.115 \\
\hline IX & 22.66 & 0.816 & 0.004 & 22.10 & 0.987 & 0.004 & 21.5 & 1.56 & 0.004 \\
\hline
\end{tabular}

It was found that in non-tumour group I, there was a statistically significant difference in volume measured depending on the planes. Median (IQR) of volume measured by axial, sagittal and coronal planes were $0.64,0.68$, and 0.58 respectively. The result shows that there was a statistically significant difference between the volume measured by coronal and sagittal planes $(\mathrm{Z}=2.563, \mathrm{P}=0.010)$ and coronal and axial plane $(\mathrm{Z}=3.090, \mathrm{P}=0.002)$. There were no significant differences between volumes measured by sagittal and coronal planes $(\mathrm{Z}=0.528, \mathrm{P}=0.598)$. Median (IQR) of volume measured for group II by axial, sagittal and coronal planes were $1.40,1.40$, and 1.26 respectively. But the median (IQR) of volume measured for group III by axial, sagittal and coronal planes were determined as 3.30, 3.00, and 3.00. The median (IQR) of volume measured for group IV were found as $4.55,4.45$, and 3.90 by axial, sagittal and coronal planes. Similarly, the median (IQR) of volume measured for group $\mathrm{V}$ shows that, axial, sagittal and coronal planes were 8.22, 9.34, and 8.80 respectively. In non-tumour group II, III, IV and V it was found that there was no significant difference between the coronal and sagittal, coronal, and axial and sagittal and axial (P $=0.472,0.097,0.165,0.197$ ). The median (IQR) of volume measured for group VI were observed by axial, sagittal and coronal planes as $10.23,9.20$, and 8.80 . But it was found that the group VI shows that there was a significant difference between coronal and axial $(\mathrm{Z}=2.742, \mathrm{P}=0.006)$. But there was no significant difference between coronal and sagittal $(\mathrm{Z}=$ $1.155, \mathrm{P}=0.248)$ and sagittal and axial $(\mathrm{Z}=0.112, \mathrm{P}=0.337)$. The median (IQR) of volume measured for group VII were determined as $16.38,15.67$, and 15.25 by axial, sagittal and coronal planes. Similarly, the group VII shows that there was a 
statistically significant difference between coronal and axial ( $\mathrm{Z}$ $=3.464, \mathrm{P}=0.001)$. There was no significant difference between coronal and sagittal and sagittal and axial $(\mathrm{Z}=1.732$, $1.731, \mathrm{P}=0.083,0.082$ ). But in the case of group VIII, there were no significant difference between the coronal and sagittal, sagittal and axial and coronal and axial planes $(\mathrm{P}=$ 0.115). The median (IQR) of volume measured for group VIII by axial, sagittal and coronal planes were 16.92, 16.03, and 16.62 respectively. But in the case of group IX there was a significant difference between coronal and axial $(\mathrm{Z}=3.300, \mathrm{P}$ $=0.001)$. It was also found that coronal and sagittal and sagittal and axial $(\mathrm{Z}=1.650,1.64, \mathrm{P}=0.099,0.098)$. For the group IX the median (IQR) volume measured were observed as 22.88 , 22.34 , and 21.60 by axial, sagittal and coronal planes.

\section{DISCUSSION}

In this hospital-based study, we investigated and analyzed the different types of non-tumour brain lesions of different dimensions in MRI images of patients. Present study revealed that, most of the non-tumour lesions like neurocysticercosis, abscess, subdural haematoma, subarachnoid haemorrhage, multiple sclerosis, cerebral ischemia, Fazekas grade 1, Fazekas grade 2, Fazekas grade 3, meningitis, cerebral infract, epidermoid cyst, arachnoid cyst, mastoiditis, cystic lesion, the axial planes are superior to the sagittal and coronal planes. Previous studies also mentioned that in general, lesions were more clearly detectable and defined on axial than on sagittal scans. ${ }^{15}$ This study also shows that the neurocysticercosis which is included in group II, the volume of MRI planes are more superior, than the volume of sagittal and coronal planes.

In one of the studies it was also well defined that the vesicular stage and intraventricular stage of neurocysticercosis is clearly given in MRI axial images. ${ }^{17}$ In this study, it was determined that the multiple sclerosis is more prominent in the volume of axial groups mentioned in group I and group VI. In another study the chronic multiple sclerosis is seen in axial images. ${ }^{15}$ Present study also mentioned that the subarachnoid haemorrhage (SAH) is observed clearer in axial plane as its volume is higher when compared to the sagittal and coronal plane. Similarly, in previous study, it was also determined that the subacute haemorrhagic infraction is also observed in MRI axial images. ${ }^{18}$

It was found that from the study, the CNS infection like abscess and neurocysticercosis which were diagnosed as clear in axial MRI imaging when compared to sagittal and coronal imaging. Previous studies also determined that in diagnosing CNS infection, MR imaging is one of the main radiological tool due to the high anatomical resolution and tissue contrast, multiplanar acquisition and high sensitivity to contrast enhancement. ${ }^{19}$ In the present studies it was found that the non-tumour brain lesions like Fazekas grade 1 (group II) and Fazekas grade 2 (group IV), the volume of lesions are observed as mass lesion when compared to sagittal plane. Similarly in one of the studies, it was found that the lesion Fazekas grade 1 and Fazekas grade 2 are observed as mild and moderate lesion in axial plane. ${ }^{20}$ From this study, it was also observed that the subdural haematoma are seen as clear massive lesion (group IX) in axial plane than other planes (sagittal and coronal). In the previous studies also, it was detected that the axial plane of subdural haematoma are seen as clear hypointense image in MRI. ${ }^{21}$ Present studies revealed that the arachnoid cyst (group IV, VII, VIII) are clearly observed in axial plane as the volume is high in axial plane as compared to the sagittal and coronal plane. In one of the studies, it was found that the large arachnoid cyst were clearly determined in axial plane when compared to the coronal and sagittal plane. ${ }^{22}$ In this study it was found that the epidermoid cyst (group VII \& VIII) were observed as well defined mass, as the volume is high when compared to other two planes (coronal and sagittal). Similarly, in another study, it was also revealed that the epidermoid cyst in MRI findings were reported as a well-defined mass in axial plane when compared to coronal and sagittal planes. ${ }^{23}$

This study also shows that, the cerebral ischemia (group II) were more in volume in axial plane when compared to coronal and axial plane. In the previous studies also, it was found that the axial MRI imaging shows two well defined small lesions. ${ }^{24}$ Mastoiditis (group VI, VII, VIII) were determined as clear mass lesion in axial images as their volume is high in axial images compared to coronal and sagittal images. In one of the studies shows that the lesion mastoiditis was observed as an enhanced image on axial MRI plane than the coronal plane. ${ }^{25}$ Present studies revealed that the cystic lesions (group VI \& VII) were observed as a well-defined mass in axial plane. But in one of the studies, it was found that the cystic lesions were well defined in both axial and sagittal plane. ${ }^{26}$

\section{CONCLUSIONS}

The advanced techniques of magnetic resonance imaging used in medical investigation has provided a huge forward leap in the field of diagnosis, particularly with avoidance of exposure to potentially dangerous ionizing radiation. This technique is safer, less expensive and moreover has better availability, so the use of MRI is becoming ever more pervasive throughout clinical practice. MRI imaging modality and its multiple applications can be used for the benefits of further informing clinical decision-making. In this article, the importance of MRI planes in diagnosis were studied and found that, in most of the non-tumour brain lesions, the volume in axial planes is more superior than sagittal and coronal planes which will help for proper diagnosis of brain lesions and further treatment.

Data sharing statement provided by the authors is available with the full text of this article at jemds.com.

Financial or other competing interests: None.

Disclosure forms provided by the authors are available with the full text of this article at jemds.com.

The authors express their appreciation and gratitude to Dr. Manju. L, Associate Professor, Department of Community Medicine, Sree Gokulam Medical College and Research Foundation for her kind support in this study for statistical evaluation and interpretation.

\section{REFERENCES}

[1] Jacobs MA, Ibrahim TS, Ouwerkerk R. MR imaging: brief overview and emerging applications. Radio Graphics 2007;27(4):1213-29. 
[2] Zivadinov R, Banas AC, Yella V, et al. Comparison of three different methods for measurement of cervical cord atrophy in multiple sclerosis. AJNR Am J Neuroradiol 2008;29(2):319-25.

[3] Villanueva-Meyer JE, Mabray MC, Cha S, et al. Current clinical brain tumor imaging. Neurosurgery 2017;81(3):397-415.

[4] Gates AR, Huang CL, Crowley JJ, et al. Magnetic resonance imaging planes for the 3-dimensional characterisation of human coronary arteries. J Anat 1994;185(Pt 2):335-46.

[5] Nayak BK, Desai S, Maheshwari S. Interpretation of magnetic resonance imaging of orbit: Simplified for ophthalmologists (Part I). J Clin Ophthalmol Res 2013;1(2):29-35.

[6] Onwu OS, Dada OM, Awojoyogbe OB. Physics and mathematics of magnetic resonance imaging for nanomedicine: an overview. World J Transl Med 2014;3(1):17-30.

[7] Bitar R, Leung G, Perng R, et al. MR pulse sequences: what every radiologist wants to know but is afraid to ask. Radiographics 2006;26(2):513-37.

[8] Pooley RA. Fundamental physics of MR imaging. RadioGraphics 2005;25(4):1087-99.

[9] McGowan JC. Basic principles of magnetic resonance imaging. Neuroimag Clin N Am 2008;18(4):623-36.

[10] Smitha H, Devi VNM, Jacob V, et al. Evaluation of signal intensity for differential diagnosis of brain lesions in magnetic resonance imaging. International J of Scientific and TechnologyResearch 2020;9(4):2840-4.

[11] Longmore DB. The principles of magnetic resonance. British Medical Bulletin 1989;45(4):848-80.

[12] Park JS, Chung MS, Park HS, et al. A proposal of new reference system for the standard axial, sagittal, coronal planes of brain based on the serially-sectioned images. J Korean Med Sci 2010;25(1):135-41.

[13] Mahmoudzadeh AP, Kashou NH. Interpolation-based super-resolution reconstruction: effects of slice thickness. J Med Imaging 2014;1(3):034007.
[14] Karpagam V, Vadanika V. Brain space occupying lesions by magnetic resonance imaging: a prospective study. International Journal of Scientific Study 2015;3(8):123-7.

[15] Rajasekaran KA, Gounder CC. Advanced brain tumor segmentation from MRI images. Intech Open 2018:83107.

[16] Kanazawa T, Ohkubo M, Kondo T, et al. Improved wedge method for the measurement of sub-millimeter slice thicknesses in magnetic resonance imaging. Radiol Phys Technol 2017;10(4):446-53.

[17] Venkat B, Aggarwal N, Makhaik S, et al. A comprehensive review of imaging findings in human cysticercosis. Jpn J Radiol 2016;34(4):241-57.

[18] Kaddah RO, Khalil ME. MR Spectroscopy evaluation of white matter signal abnormalities of different nonneoplastic brain lesions. Egyptian J Radiol Nucl Med 2016;47(1):233-42.

[19] Cuvinciuc V, Vargas MI, Lovblad KO, et al. Diagnosing infection of the CNS with MRI. Imaging in Medicine 2011;3(6):689-710.

[20] Marek M, Horyniecki M, Frączek M, et al. Leukoaraiosis new concepts and modern imaging. Pol J Radiol 2018;83:e76-81.

[21] Fobben ES, Grossman RI, Atlas SW, et al. MR characteristics of subdural hematomas and hygromas at 1.5 T. AJNR 1989;10:687-93.

[22] Di Gaeta A, Giurazza F, Guarnieri G, et al. Giant arachnoid cyst associated with acute subdural haematoma: a case report. Neuroradiol J 2017;30(3):286-9.

[23] Hoang VT, Trinh CT, Nguyen $\mathrm{CH}$, et al. Overview of epidermoid cyst. Eur J Radiol Open 2019;6:291-301.

[24] Choi PMC, Ly JV, Srikanth H, et al. Differentiating between Hemorrhagic Infarct and Parenchymal Intracerebral Hemorrhage. Radiol Res Pract 2012;2012:475497.

[25] Platzek I, Kitzler HH, Gudziol V, et al. Magnetic resonance imaging in acute mastoiditis. Acta Radiol Short Rep 2014;3(2):2047981614523415.

[26] Hingwala DR, Radhakrishnan N, Kesavadas C, et al. Neuroenteric cysts of the brain-comprehensive magnetic resonance imaging. Indian J Radiol Imaging 2013;23(2):155-63. 